\title{
Studies on Plasma and Plasma Waves in the Plasmasphere and Auroral Particle Acceleration Region, by PWS on board the EXOS-D (Akebono) Satellite
}

\author{
Hiroshi OYA \\ Department of Geophysics and Astronomy, Tohoku University, Sendai 980. Japan
}

(Received January 5, 1991; Revised August 7, 1991)

\begin{abstract}
Plasma wave and sounder experiments (PWS) on board the EXOS-D (Akebono) satellite are continuously providing the wide variety of data which contribute to the studies on plasma wave phenomena associated with the wave particle interactions in the regions of the cusp, auroral particle acceleration, and magnetic equator. Observation results of the auroral kilometric radiation (AKR) with Poynting vector, polarization as well as the dynamic spectra, have indicated that the generation mechanism of AKR is not necessarily due to the cyclotron maser mechanism but majority parts of AKR's are caused by the conversion of upper hybrid waves through the Doppler conversion processes and also through irregularities of the plasma in the auroral particle acceleration regions. Propagating electromagnetic wave phenomena in the plasmasphere are 1) banded rising and falling LF waves (BRIFLE), 2) discrete $\mathrm{HF}$ emissions, and 3) terrestrial hectometric radiations. There are localized modes of the plasma waves such as 4) upper hybrid mode waves, 5) Z-mode waves and 6) quasielectrostatic whistler mode waves. Reflecting the evidence of the strong wave particle interactions, in the region of the cusp and auroral particle precipitation region, 7) broad band electrostatic noises are frequently observed and these become good indicator of the particle precipitation when the low energetic particle detector makes a pause of their operation in the low latitude range. Storm time effects of the plasma waves show also unique and important features; plasmaspheric odd harmonic, $(n+1 / 2) f_{c}$ emissions which have never detected in the plasmasphere inside of the plasmapause in the regular condition of the magnetic field activities take place even in the plasmasphere inside of the plasmapause in the times of large magnetic storm.

The plasma density profiles obtained from the observed upper hybrid waves provide wide variety of variation of the plasmasphere and plasmapause structure with informations of the plasma instabilities there. A review has been made for the instabilities identified in the magnetic equator whose occurrence region is circulating at the magnetic equator covering the altitude from $1,000 \mathrm{~km}$ to $10,000 \mathrm{~km}$. In the storm time, the plasmapause has apparently been disrupted with depressed density and loosing sharp edge of the density variation. In the recovery phase of the large magnetic storm, the plasma density distribution shows large disturbances where the amplitudes of the irregular variation of the plasma density become 5 to 6 times or sometimes an order of magnitude of the background density itself. Also during recovery phase of the storm, a large scale cavity of the plasma is formed centered at the magnetic equator coinciding with the position of expected ring current. All of these results show that the PWS on board the EXOS-D satellite are making effective contribution to study on the energy transfer through the plasmasphere providing data of the electron density, temperature anisotropy and informations on existing free energy of the plasma and the energetic particles.
\end{abstract}


1. Introduction

After successful launching of the Akebono(EXOS-D) satellite, on Feb. 22, 1989, the turns on of the science instrument have effectively been made for the on board eight science instruments:

1) Magnetic Field Detector (MGF)

2) Electric Field Detector (EFD)

3) Plasma Wave and Sounder Experiments (PWS)

4) Very Low Frequency Plasma Wave Detector (VLF)

5) Low Energetic Particle Detector (VLF)

6) Suprathermal Mass Spectrometer (SMS)

7) Temperature and Energy Detector for Electron (TED) and

8) Auroral Television Camera (ATV).

Turn on of the waves and field experiments has been made early March 1989, and the turn on of the instruments using high voltage power sources has been made early April 1989.

In addition to main 8 science experiments, an instrument for radiation belt monitor in an energy range from $0.5 \mathrm{MeV}$ to $3 \mathrm{MeV}$ has also been installed. The details of the instrumentations have already been published with initial results (Special issue of JGG, No. 4, 1990 and GRL, Vol. 18, No. 2, 1990).

Though the initially designed life time of Akebono satellite is 1 year from the engineering analyses stand point, the satellite is expected to continue the observations during 5 year. The operation of the satellite will therefore contribute to the early parts of STEP projects, the world most extensively organized program of SCOSTEP for the solar terrestrial physics in 1990's.

In these widely integrated observations, the present paper concentrates on the observation results of PWS (Plasma Wave and Sounder Experiments); from a variety of the results, we will select here subjects directly related to the plasma wave phenomena in high frequency range (higher than $20 \mathrm{kHz}$ ) and also phenomena related to the plasma dynamics based on the plasma density deduced from the observed upper hybrid wave emission frequency. The region of the present studies are then divided into

1) Plasma wave phenomena relating to the wave-particle interactions,

2) Polar regions,

3) Equatorial region plasmasphere, and

4) Plasmasphere structure.

During early phase of the EXOS-D (Akebono) satellite, three major magnetic storms had taken place; i.e., in March 13 to 14th, Oct. 20 and 21st and Nov. 17 and 18th, in 1989. These data of large magnetic storms have been associated with evidences that have never been disclosed by the previous observation, in polar region, plasmasphere, and equatorial plasmasphere. Observations of these evidences become important keys to solve the hidden question of dynamics and energy transfer through the plasmasphere and polar region plasmas. The contents of the present paper, then, consist of reports on the newly discovered evidences including the review of the already reported new finding from PWS on board the EXOS-D satellite. 


\section{Plasma Waves Observed by the EXOS-D Satellite}

Plasma wave phenomena observed by the EXOS-D satellite have been summarized by adding already found phenomena (OYA et al., 1990), as

I) Propagating electromagnetic waves;

1) Solar type III radio bursts,

2) Auroral kilometric radiations (AKR),

3) Banded rising and falling LF waves (BRIFLE),

4) Discrete HF emission,

5) Terrestrial hectometric radiation (THR).

II) Quasi-propagating mode waves;

1) Upper hybrid mode waves,

2) Z-Mode waves,

3) Quasi electrostatic whistler mode waves.

III) Insitu electrostatic plasma waves;

1) Plasmaspheric odd harmonic emissions $(n+1 / 2) f_{c}$,

2) Broad band electrostatic noises.

In the above items, auroral kilometric radiations (AKR) belong to the subject which is considered with special interest in the field of the studies on the auroral processes and substorms activities, also in the field of the origin of the auroral particle acceleration. AKR will, then, be described separately in the next section.

The observations of the type III radiation using the satellite have been made the start with the ATS-II satellite for the frequency range of $3.0 \mathrm{MHz}$ to $450 \mathrm{kHz}$ (ALEXANDER et al., 1969), then observation study of long wave type III radio burst has been expanded by using OGO-III (GRAEDEL, 1970), and by Radio Astronomy Explorer (RAE-1) satellite (MOHR et al., 1969; EVANS et al., 1971), OGO-5 and IMP-6 (FRANK and GURNETT, 1972; EVANS et al., 1973; ALVAREZ et al., 1972 and 1975; KAISER, 1975). Discussions have been raised for the generation mechanism of type III radio bursts; whether the source is fundamental frequency of the Langmuir waves or the second harmonics of the generated waves, which are formed as the results of the nonlinear coupling processes. To clarify these points many observation studies have also been continued. One of these efforts is to identify the source position of the type III radio bursts by multi satellite observations (WEBER et al., 1977; GURNETT et al., 1978). ISEE-3 (LIN et al., 1981) and also Voyager (SAWYER and WARWICK, 1987) have made contribution to study the energetic particle effects through the interplanetary space generating the kilometric range type III radio bursts. To studies the generation mechanism and also interaction processes of the energetic electron through the interplanetary media, EXOS-B (Jikiken) data have also made contribution (MrYAKE and OYA, 1989). By the EXOS-D satellite large volume of data of the solar type III radio bursts, in the frequency range from $5 \mathrm{MHz}$ down to $20 \mathrm{kHz}$ are obtained; especially in the time of active solar surface conditions very frequent occurrence with rate of every $30 \mathrm{sec}$ (in the case of maximum) has been identified. These data of type III radio bursts will contribute to future studies on the origin of the type III bursts in the interplanetary space.

\subsection{Banded rising and falling LF waves (BRIFLE)}

The special dynamic spectrum feature of radiation in the frequency range of LF $(20 \mathrm{kHz}-$ $1 \mathrm{MHz}$ ) has first been identified by the PWS on board the EXOS-D satellite (OYA et al., 1991; KIKUCHI et al., 1991). The banded spectra of these newly identified emissions show the 
rising and falling variation with the maximum frequency range at the magnetic equator, and named the waves BRIFLE (Banded Rising and Falling LF Emission).

An example of BRIFLE is given in Fig. 1. The minimum frequency of BRIFLE is always identified in a range of $L=1.2-1.6$; and the theoretical studies (KIKUCHI et al., 1991) show that the BRIFLE's are the phenomena of the propagating whistler mode waves, in the LF frequency range, which are generated at $L=1.2$, to 1.6 regions in the relatively higher altitude about $1000 \mathrm{~km}$. Detail description will be given in another paper (OYA et al., 1991; KIKUCHI et al., 1991).

\subsection{Discrete HF emissions}

In the frequency range above $100 \mathrm{kHz}$, emissions with discrete flat nature has been observed. An example related to the discovery of these phenomena is reproduced in Fig. 2 (OYA et al., 1990). The phenomena apparently show that the origin of the HF emissions is associated with the large density irregularities; and the origin of this discrete emissions is in the conversion processes of the plasma waves into electromagnetic waves. (OYA, 1971a, 1974; JONES, 1976; OKUDA et al., 1982).

\subsection{Terrestrial hectometric radiations (THR)}

THR has been discovered by PWS on board the EXOS-C (OYA et al., 1985); and has also been observed by the EXOS-D satellite (OYA et al., 1990). The PWS on board the EXOS-D will make important contributions for studies on the generations of THR for its mechanism and relation to the auroral activities and even for the similarity of the generation with Jovian decametric wave radiations.

\subsection{Upper hybrid mode waves}

In early part of the studies on the upper hybrid mode waves, WALSH et al. (1964); HUGUENIN et al. (1964); HARVEY (1965); and BAUER and STONE (1968) have made important contribution. BAUER and STONE (1968) had provided more complete report of the upper hybrid emissions based on the ATS II satellite observations in the altitude range about $1,000 \mathrm{~km}$ to $10,000 \mathrm{~km}$. In parallel to these reports, there are also several important reports for existence of the upper hybrid mode waves (HARVEY, 1968; GREGORY, 1969; MOISIER et al., 1973). For the generation mechanism, Cerenkov radiation in the form of electromagnetic waves in cold plasma had been proposed (WALSH et al., 1964; BAUER and STONE, 1968; GREGORY, 1969; MULDREW, 1970; LAASPERE et al., 1971). Importance of the upper hybrid mode waves as the channel which connect between the electrostatic plasma waves and the Z-mode electromagnetic waves, had first been pointed out by OYA (1971a) based on the full wave treatment of the dispersion relation; and also it is pointed out that the upper hybrid mode waves have possibility to be generated making resonance with beam of very wide range of the energy. Studies on the upper hybrid mode waves have also been continued (LIM and LAASPERE, 1972; MOISIER et al., 1973; TAYLOR and SHAWHAN, 1974; MAGGS, 1978; KURTH et al., 1979 and 1981).

Detail points of the new observation results of upper hybrid mode waves obtained by the EXOS-D satellite have already been published (OYA et al., 1990, 1991); we will not then repeat here for this subject. Generation processes of the upper hybrid waves are related to two complementary features of free energy; i.e., one is the case of the plasma velocity 
distributions enhanced in the perpendicular direction with respect to the magnetic field, and the another is the case of the plasma with electron or ion beams.

Because PWS on board the EXOS-D satellite has the capability to detect even very weak upper hybrid mode waves (the threshold is $0.1 \mu \mathrm{V} / \mathrm{m}$ ) we can always detect the waves and can use the detected UHR frequency $f_{\mathrm{UHR}}=\sqrt{f_{\mathrm{p}}^{2}+f_{c}^{2}}$, where $f_{\mathrm{p}}$ and $f_{\mathrm{c}}$ are, respectively, plasma frequency and the electron cyclotron frequency, to deduce the precise plasma density distributions.

\section{$2.5 \mathrm{Z}$-mode waves}

After pointing out of the existence of the naturally generated Z-mode waves in space (OYA and MORIOKA, 1983) evidences and theories for Z-mode waves are reported (JONES, 1984; OMIDI et al., 1984; DUSENBERY and LYONS, 1985; OMIDI and WU, 1985; MENIETTI, 1985; BAHNSEN et al., 1987; HASHIMOTO et al., 1987; HORNE, 1988; MORIOKA et al., 1988; HORNE, 1989; HASHIMOTO and CALVERT, 1990). As has been given in the example given in Fig. 3a very remarkable existence of the Z-mode waves are frequently identified by the

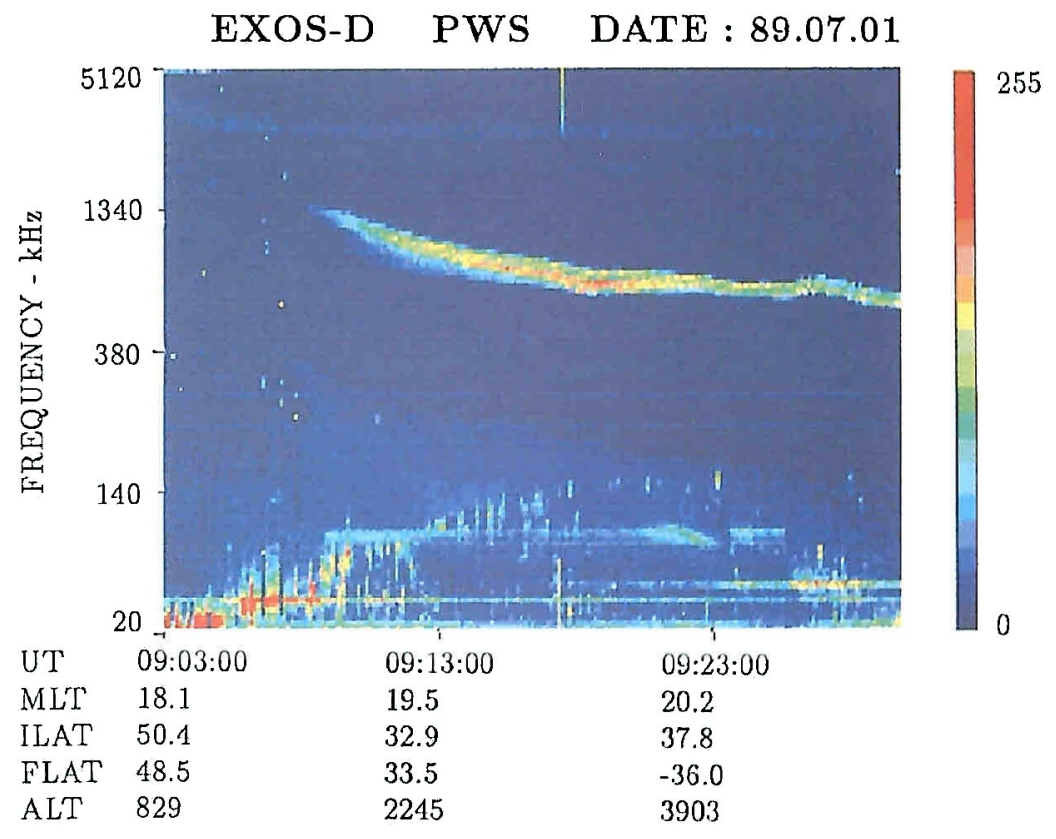

Fig. 1. Example of BRIFLE, in the dynamic spectrum of the PWS on board the EXOS-D satellite. The ordinate of the diagram shows the frequency from $20 \mathrm{kHz}$ to $5 \mathrm{MHz}$ with abscissa which indicates the observation time in UT., and Satellite position in MLT (magnetic local time), ILAT (invariant latitude), FLAT (Foot latitude of dipole magnetic field), and ALT (altitude) in $\mathrm{km}$ at corresponding observation time. The intensity is indicated by color code for relative level from 0 to 255 . The faint emission below local electron cyclotron frequency $f_{c}$, which show the banded spectra whose center frequency makes continuous variation with maximum at the magnetic equator is BRIFLE. 
EXOS-D, PWS data. It is also clarified that even no separate signature has been recognized in the dynamic spectra of PWS (see Fig. 3b), Z-mode waves are always associated with the existing upper hybrid mode waves, as we can see in Fig. 3c, where the Z-cutoff frequency is identified very close to the upper hybrid frequency because of the condition $f_{\mathrm{p}} \gg f_{\mathrm{c}}$.

\subsection{Quasi electrostatic whistler mode waves}

It is well known that the whistler mode waves are naturally connected to the electrostatic plasma wave modes when the wavelength makes change while the waves propagate through inhomogeneous media. Between the electrostatic plasma waves and electromagnetic Z-mode waves there is a regime of the quasi-electrostatic whistler mode waves. The frequency range of this quasi electrostatic plasma waves is almost identical with the resonance; i.e.,

$$
\begin{gathered}
2(1-X)-Y^{2} \sin ^{2} \theta-\sqrt{Y^{4} \sin ^{4} \theta+4 Y^{2}(1-X)^{2} \cos ^{2} \theta}=0, \\
X=\frac{f_{\mathrm{p}}^{2}}{f^{2}}, \quad Y=\frac{f_{\mathrm{c}}}{f},
\end{gathered}
$$

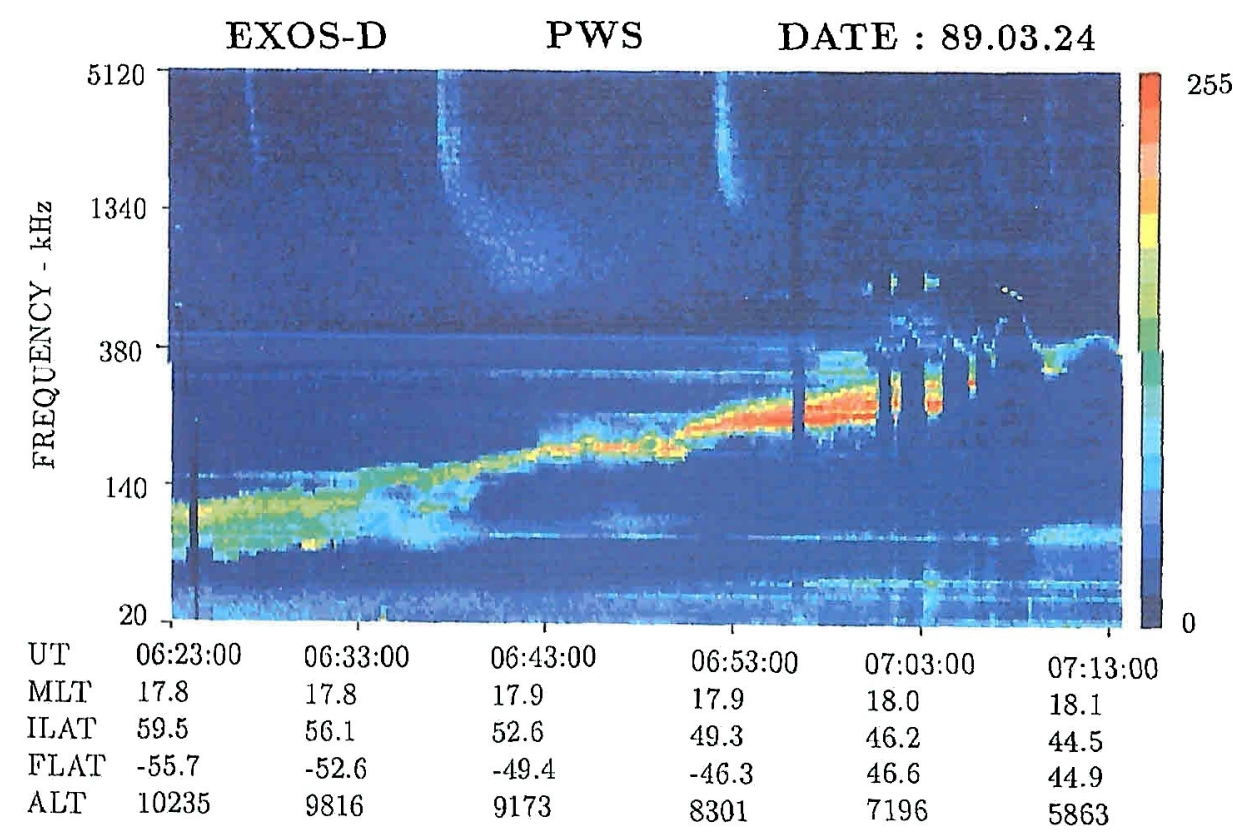

Fig. 2. Example of the discrete HF emissions observed on March 24, 1989. The dynamic spectrum is indicated with the same format as Fig. 1; straight line shape (except for a disturbance at $500 \mathrm{kHz}$ ) of the emission which merges at the local upper hybrid waves at 06:33 UT and 07:03 UT are the discrete HF emissions that are understood as the manifestation of the conversion of the upper hybrid waves into electromagnetic waves (After OYA et al., 1990). 


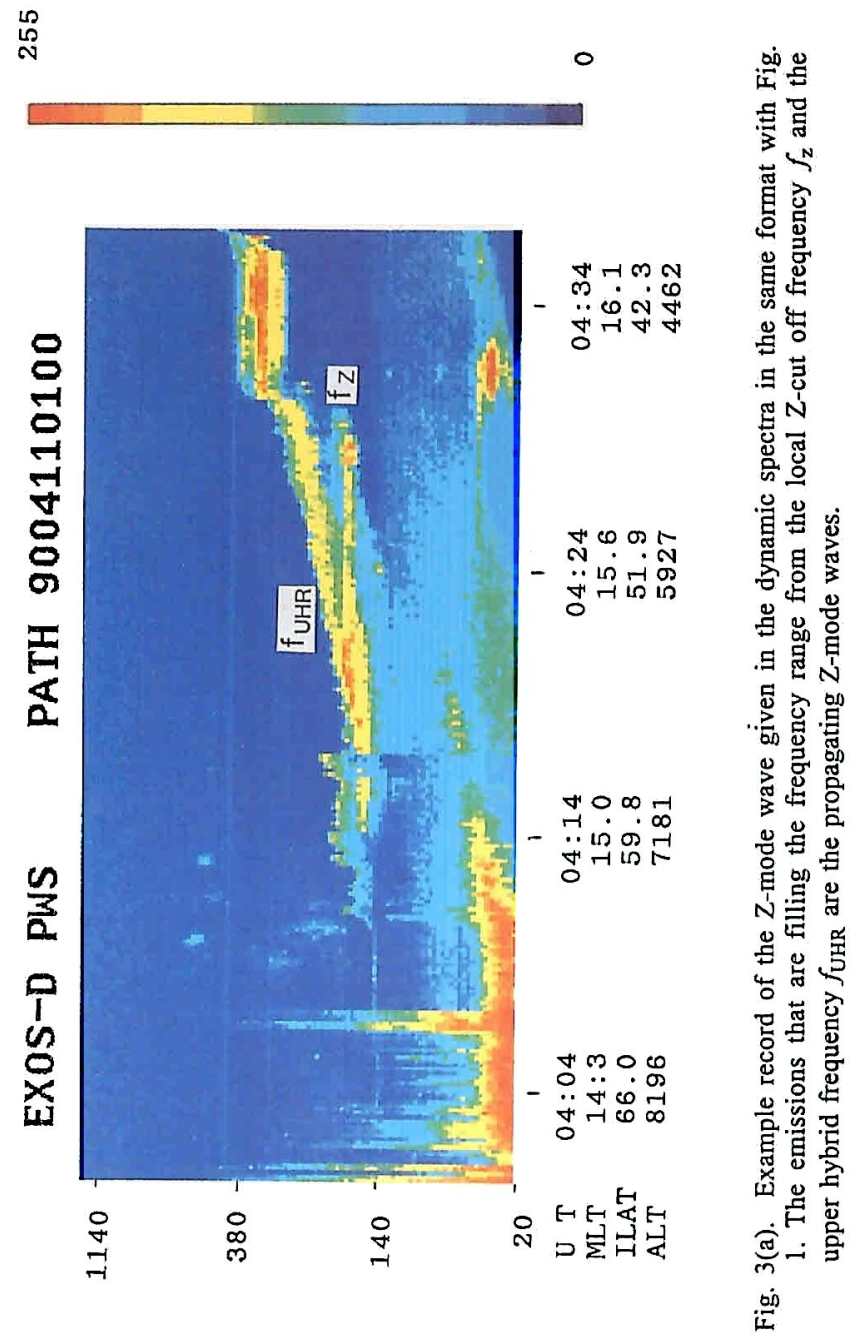

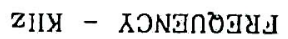


in the frequency range $f<\operatorname{Min}\left(f_{\mathrm{p}}, f_{\mathrm{c}}\right)$ where $\operatorname{Min}\left(f_{\mathrm{p}}, f_{\mathrm{c}}\right)$ shows lower frequency between $f_{\mathrm{p}}$ and $f_{\mathrm{c}}$. Therefore, many of the propagating mode waves are identified as the regular whistler mode waves but when the satellite is passing through the region of the particle precipitations, the mode is taken to be electrostatic or quasi electrostatic plasma wave mode. One typical example of these phenomena is identified in the time of the large magnetic storm

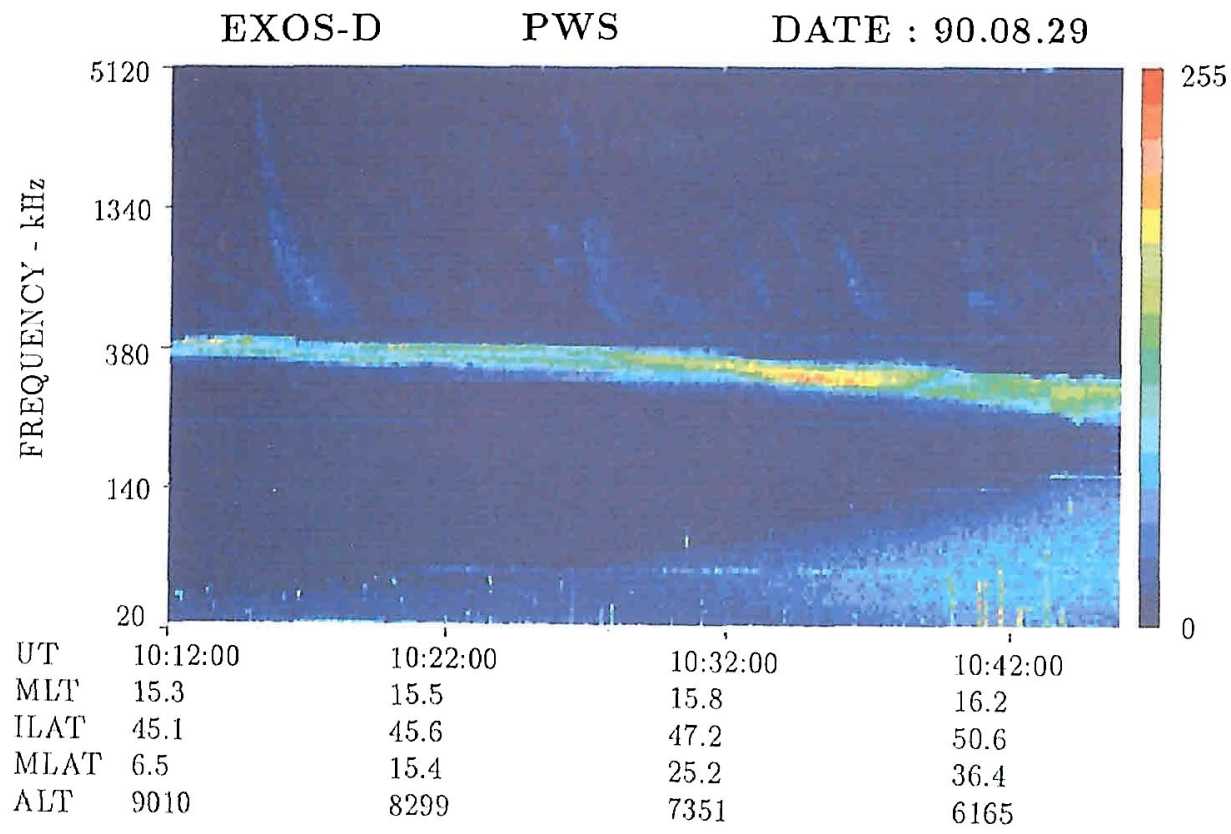

Fig. 3(b). Example of the dynamic spectrum observed on August 29, 1990 in same format with Fig. 1.

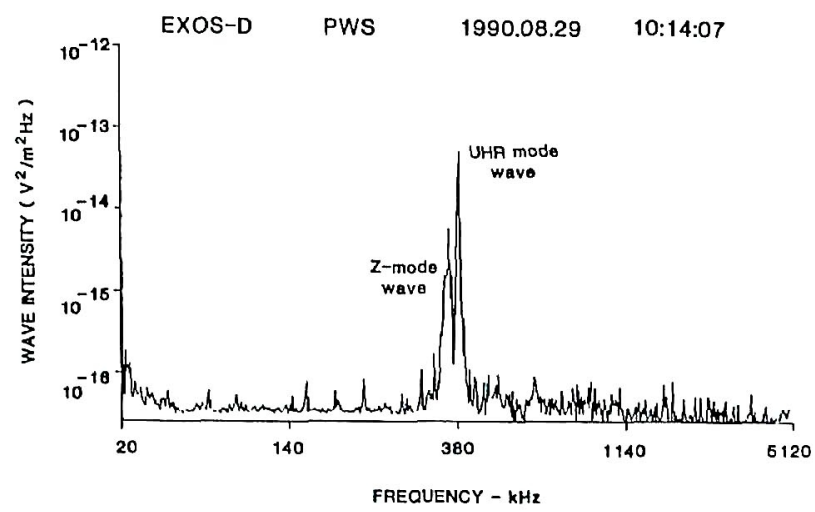

Fig. 3(c). The spectrum of $\mathrm{Z}$-mode wave which is located very close to the spectrum of the upper hybrid mode waves because $f_{U H R}$ is very close to $f_{\mathrm{z}}$ for the condition of $f_{\mathrm{p}} \gg f_{\mathrm{c}}$. 
as given in Fig. 4 where intense quasi static plasma waves can be identified below the local plasma frequency $f_{\mathrm{p}}$ because $f_{\mathrm{p}}<f_{\mathrm{c}}$.

\subsection{Plasmaspheric odd harmonic $(n+1 / 2) f_{c}$ emissions}

The first report of the odd harmonic emission had been made by KENNELL et al. (1970) based on the observations of the OGO-5 satellite in the magnetospheric plasma sheet region. Almost in parallel, the diffuse resonance $f_{\mathrm{Dn}}$ has been identified being based on the Alouette II data; the resonance is tightly connected to the $(n+1 / 2) f_{\mathrm{c}}$ emissions though the phenomena also involve the nonlinear plasma wave effects (OYA, 1970, 1971b). After these report there were many research works for this $(n+1 / 2) f_{c}$ emissions.

The present result belongs, however, to new subject because the plasmaspheric odd harmonics $(n+1 / 2) f_{c}$ waves have first identified within the plasmasphere by the EXOS-D satellite observation. An example record is given in Fig. 5 where the $(n+1 / 2) f_{\mathrm{c}}$ emissions are indicated through whole the range of the satellite path in the plasmasphere inside of the plasmapause in the time of the large magnetic storm which started from 01:30 UT, on March 13,1989 . It has well been established that the $(n+1 / 2) f_{\mathrm{c}}$ emissions are caused by the free energy state where the velocity distribution of particles is enhanced in the direction perpendicular to the magnetic field (FREDERICS, 1971; OYA, 1971b; KARPMAN et al., 1973; YOUNG et al., 1973). The existence of $(n+1 / 2) f_{\mathrm{c}}$ emissions in the plasmasphere, therefore,

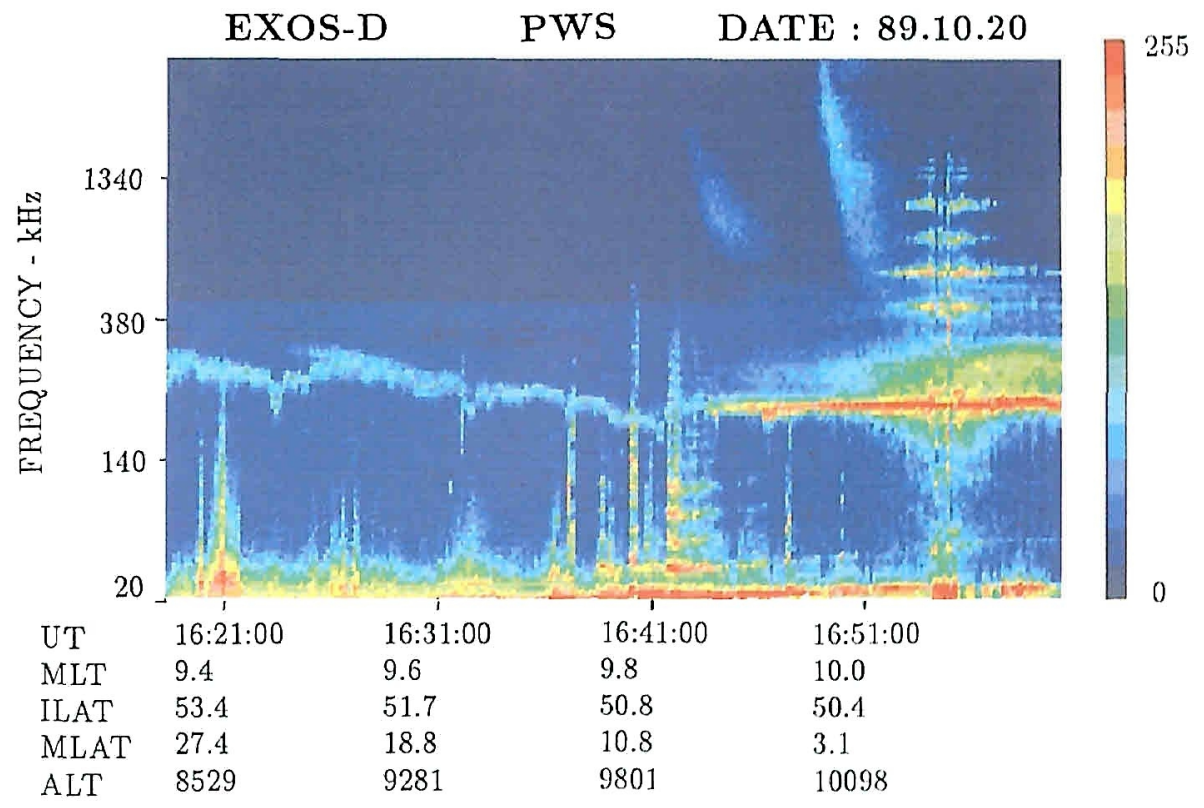

Fig. 4. PWS data observed on Oct. 20, 1989 in the main phase of the large magnetic storm. The format of the diagram is same with Fig. 1. Below the upper hybrid frequency there are widely distributed broad band emission that show vertically structured emission spectra. Below the electron cyclotron frequency there is a fairly wide band trend of the emissions which are identified as the quasi electrostatic whistler mode waves. 


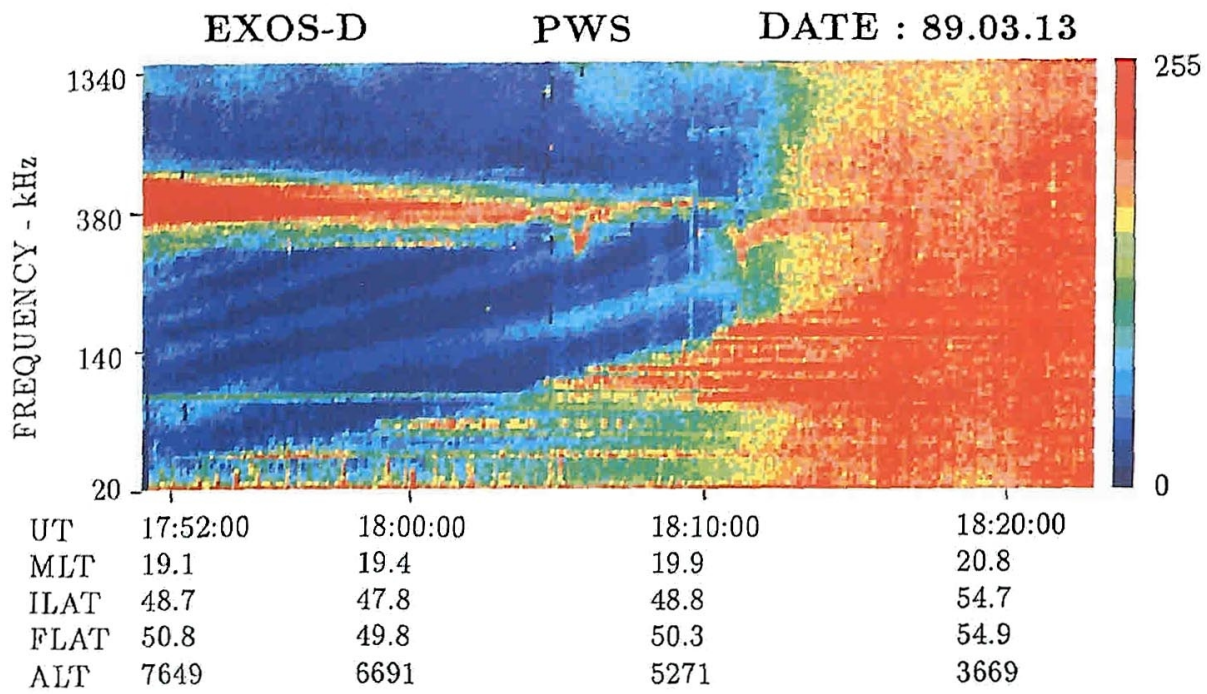

(a)

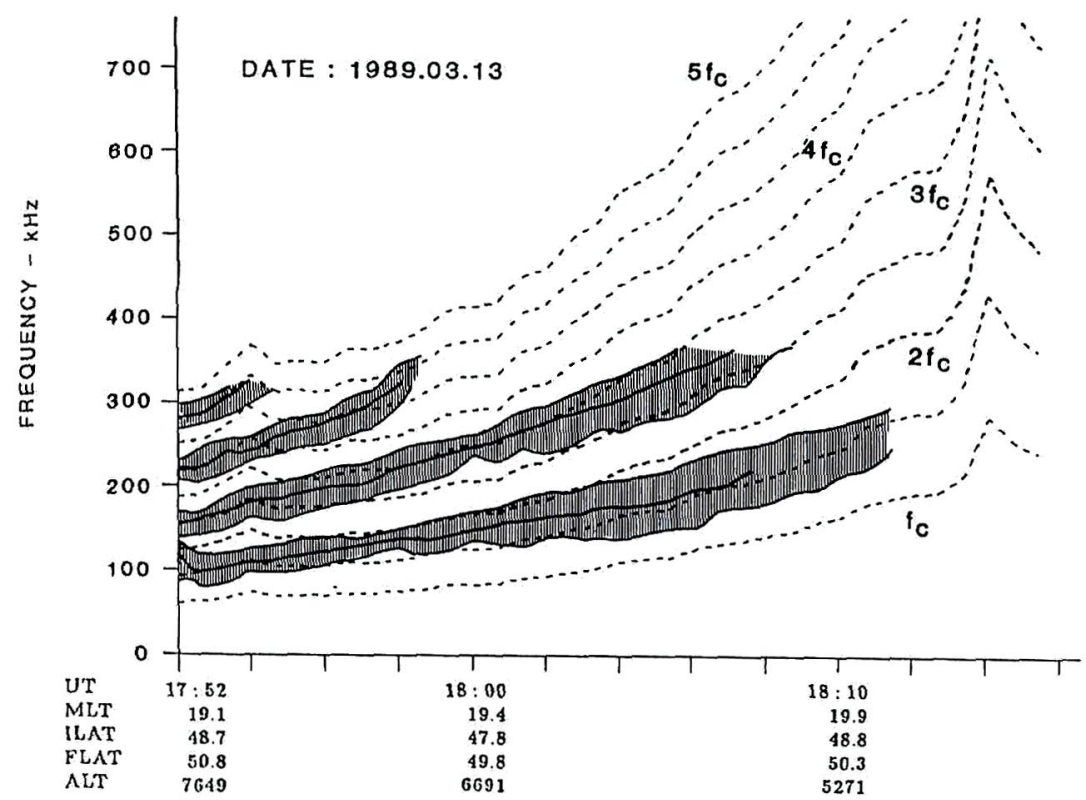

(b)

Fig. 5. (a) The plasmaspheric odd harmonic, $(n+1 / 2) f_{\mathrm{c}}$ emissions observed on March 13, 1989 in the main phase of the large magnetic storm. The observed dynamic spectrum is indicated in the same format with Fig. 1. (b) The same case with (a) is expressed with scaling by the electron cyclotron frequency $f_{\mathrm{c}}$. 


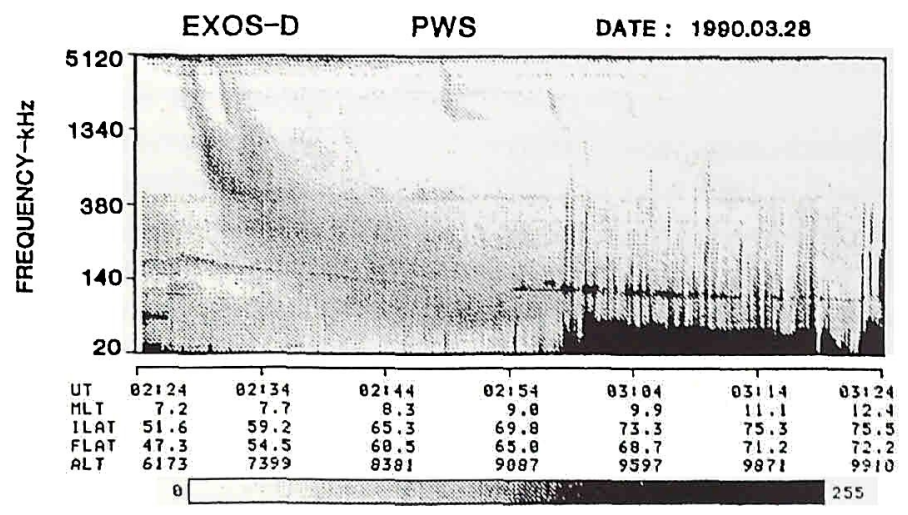

Fig. 6. Typical example of the broad band electrostatic noise observed in the cusp region on March 28, 1990. The dynamic spectrum is given in the same frame with Fig. 1, but the intensity is indicated by gray code.

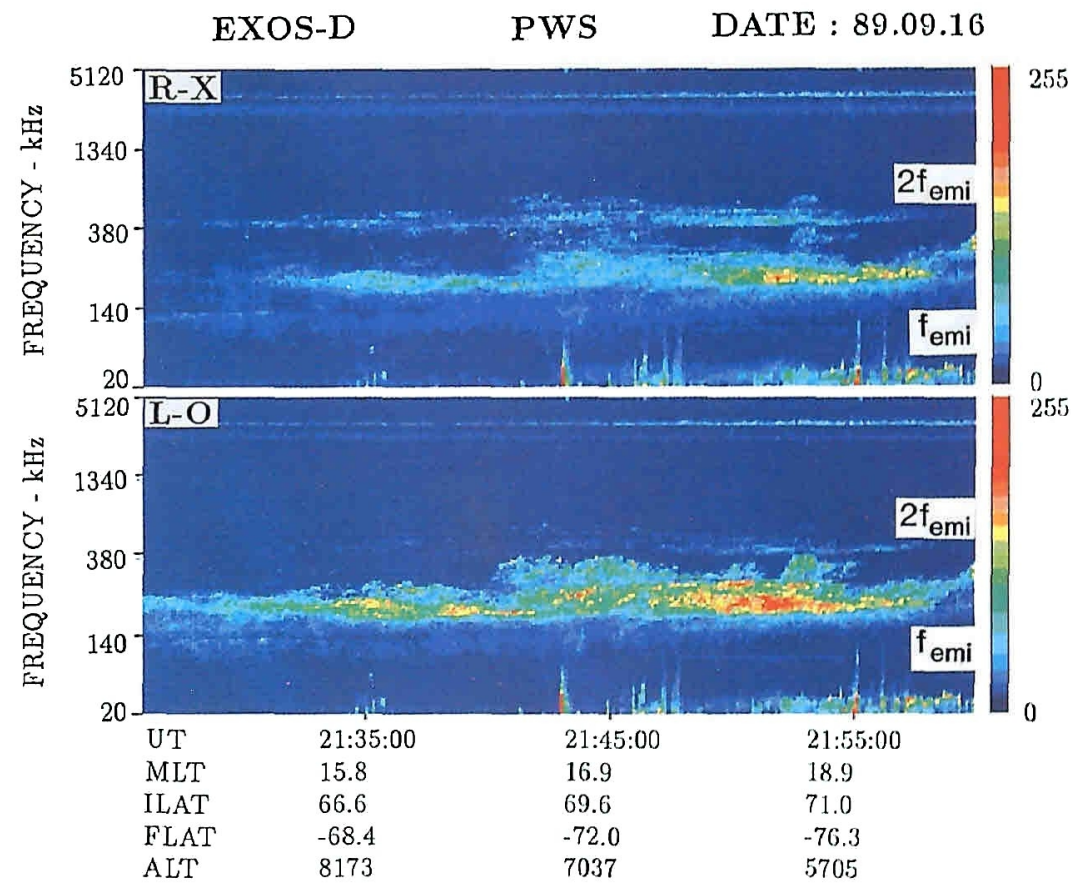

Fig. 7. Example of the discrete $A K R$ which shows the harmonic relation. The dynamic spectra are displayed for the two polarizations, wave modes are identified to be R-X (top) and L-O (bottom) when the waves come from the source in the anti-sun ward direction. Format for each panel is same with Fig. 1. The data show that the relative intensities of the left hand to the right hand polarized components for the case of the 2 nd harmonics $2 f_{\mathrm{cmi}}$ are completely opposite to the case of the fundamental $f_{\text {emi }}$ where $f_{\text {emi }}$ indicate the emission frequency (after OYA, 1990). 
indicates the evidence of the large free energy injection in the plasmasphere in the time of the large magnetic storms.

\subsection{Broad band electrostatic noise (BEN)}

The phenomena of BEN have first been reported by GURNETT et al. (1976), In the region of the auroral particle precipitation, the electrostatic broad band noises have been identified using the DE-1 satellite data (LIN et al., 1989) and the BEN phenomena have also been identified in the region of the cusp in the height close to the ionosphere by using Viking data (POTTELETTE et al., 1990). The phenomena are very sensitive indicator of the existence of the beam injected through the plasma. As given in Fig. 6, we can see that the broad band emissions are closely associated with the cusp region particle precipitation where large flux of soft electrons are injected.

One of the important new evidences of the observation of the broad band emission which is not necessarily related to the cusp particle precipitation is the case of low latitude precipitation in the time of the large magnetic storms. As has been given also in Fig. 4, there are widely distributed area of BEN in lower latitude than usual cusp and also the auroral particle precipitation regions; the region of the occurrence of BEN is expanded even close to the equatorial region. There are largely enhanced region of broad band emissions in low latitude range suggesting the existence of large flux precipitation of the soft energetic electron even in middle and low latitude regions, in the time of the large magnetic storms.

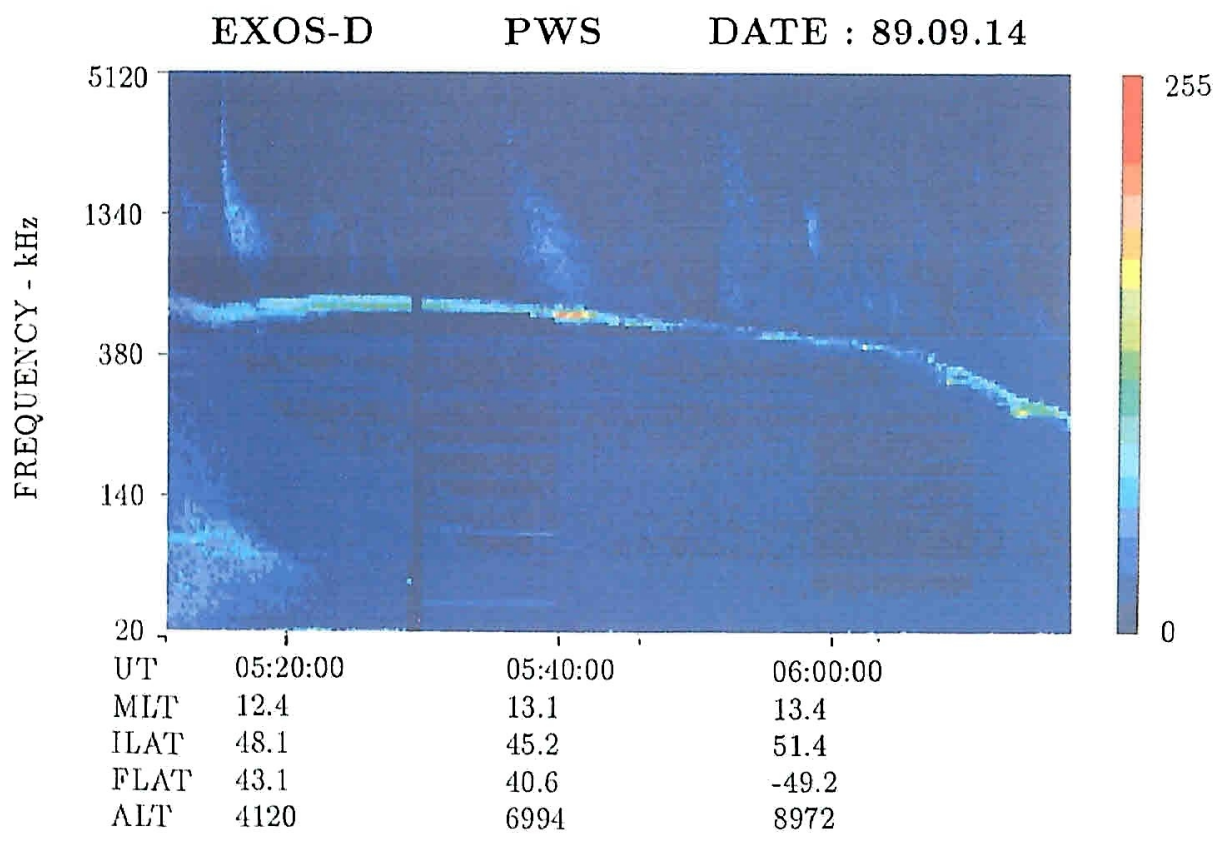

Fig. 8. An example of EPWAT phenomenon observed on Sept. 14, 1989 in the period of 05:10 to 06:20 UT; the format of the diagram is the same with Fig. 1. In the trend of the upper hybrid mode waves, there is a very intensified portion when the satellite passed through the magnetic equator. 
3. Auroral Kilometric Radiations to Detect the Acceleration Region of Auroral Particles

For the studies on the auroral particle acceleration and polar region phenomena, observations of AKR phenomena by PWS on board the EXOS-D satellite are making one of the most remarkable contribution.

After suggestions of the noises from the polar region (BENEDIKTOV et al., 1965; DUNKEL et al., 1970; STONE, 1973), GURNETT (1974) has made first identification of the terrestrial kilometric radiation which is generated in connection with the auroral phenomena. The observational evidences (KAISER and STONE, 1975; KURTH et al., 1975; GREEN et al., 1977; VOOTS et al., 1977; GALLAGHER and GURNETT, 1979; MORIOKA et al., 1981) give a clear feature of AKR as the electromagnetic wave emission generated in the region of the auroral particle accelerations which are formed in the range from $3000 \mathrm{~km}$ (BENSON and CALVERT, 1979) to $2 R_{E}$ (GURNETT, 1974) where $R_{E}$ is the earth's radius.

Because the generation of AKR is directly related to the acceleration of the particles, the detailed investigation of AKR provides important informations for the environment to generate AKR associated with the magnetospheric sub-storm activities. To understand the back ground condition of AKR generation, however, precise emission mechanism should be clarified for the origin of AKR. The presently well known theory is the cyclotron maser mechanism, original back ground of this theory has been proposed by WU and LEE (1979). In their theory, the most important and unique point is in the resonance condition where they proposed the relativistic situation as

$$
\omega-k_{\|} V_{\|}-\Omega \sqrt{1-\beta^{2}}=0
$$

where $k_{\|}, V_{\|}, \beta$ and $\Omega$ are the wave number in parallel to the magnetic field, the particle velocity in parallel to the magnetic field, and the $V / c$ value for the total velocity of the particle $V$, and angular electron cyclotron frequency, respectively. Along this line of the resonance condition, the studies have further developed by OMIDI and GURNETT $(1982,1984)$ to cover the case of the real velocity distribution where the trapped particles makes resonance. CALVERT (1982) has proposed laser mechanism where the standing waves of AKR trapped in the cavity region of the plasma, in the acceleration of the auroral particles, make coherent tuning with incoming beam of the auroral particle.

Contrary to this relativistic cyclotron mechanism, there was the proposal of the conversion theory (BENSON, 1975) based on the theory of the generation of the Jovian decametric radiation in terms of the conversion of the electrostatic plasma waves into electromagnetic waves (OYA, 1974); afterwards, Benson has changed his stand point, to support the maser mechanism (BENSON et al., 1980). Based on the studies of the EXOS-D observations of AKR, the conversion theory has been newly proposed by OYA (1990). In the case of the EXOS-D, new results have been obtained in addition to the regular spectra observations. That is, the observations of the polarization and the Poynting vector (MORIOKA et al., 1990) provide important information for the mode of AKR at the source. The studies on the source plasma condition of AKR by using observed upper hybrid mode wave frequency and also the radio wave sounder (OYA et al., 1990) provide important key to check the theory.

The result shows that the AKR source condition has not necessarily fits with plasma conditions required by the cyclotron maser mechanism which has originally been proposed by WU and LEE (1979). The results show that the source conditions are favorable to the 
processes of conversion of electrostatic plasma waves into electromagnetic waves (OYA, 1990). That is, in the conversion studies there are three important points:

1) Observed plasma conditions in the source region of AKR in terms of the $f_{\mathrm{p}} / f_{\mathrm{c}}$ value is not necessarily less than 0.1 but very frequently over 0.3 ; i.e. the result indicates that AKR's are observed clearly even the plasma conditions for the cyclotron maser mechanism is not satisfied.

2) The polarization of AKR is thought simply to be intense R-X mode with weak L$O$ mode waves in the cyclotron maser mechanism. The studies based on the EXOS-D observation (MORIOKA et al., 1990) however reveal that the waves show L-O mode for the case of weak auroral activities and the mode is changed to the intense R-X mode with weaker $\mathrm{L}-\mathrm{O}$ mode in the case of the intense auroral activities.

3) Evidence of discrete AKR with the second harmonics has been discovered. This is very new subject whose example is reproduced in Fig. 7 where 2 nd harmonics $2 f_{\text {emi }}$ of the burst are identified with AKR emissions at the fundamental frequency $f_{\text {emi }}$. The results suggest that the source waves are generated in the form of the local upper hybrid mode waves; and these source mode of plasma waves are converted to electromagnetic waves. The fundamental waves of upper hybrid mode waves in the source region are converted through the linear conversion processes while the 2 nd harmonics are possibly converted through the nonlinear coupling processes including three wave interaction of the plasma waves at the upper hybrid mode wave frequency branch (OYA, 1990).

The evidence of polarization of AKR given in the item 2) is consistent with the Doppler conversion processes in the auroral particle precipitation regions (OYA, 1990; OYA and IIZIMA, 1991).

\section{Equatorial Region Effects-Enhancements of the Upper Hybrid Mode Waves}

One of the most remarkable new finding of the PWS experiments on board the (EXOSD) satellite is the equatorial enhancements of the upper hybrid mode waves (OYA et al., 1990), which is called EPWAT (Equatorial plasma wave turbulence). An example of EPWAT is given in Fig. 8. The regions of enhancements of the upper hybrid waves are localized in a belt circulating at the magnetic equator forming the disc region like the Saturnian ring (see Fig. 9). The EPWAT phenomena are always observable even in the time of the quiet magnetic conditions; in the intensity of EPWAT, there is however, component which is strictly related to the $\sum K p$ index.

In the period of extremely enhanced magnetic storms, the EPWAT phenomena are also enhanced in its intensity with expansion of region of the occurrence of EPWAT. The EPWAT phenomena are understood as the results of an inherent mechanism to generate free energy of the plasma in the magnetic equator region inside the plasmapause. One of the possible mechanism is inferred to be the plasma drifting across the magnetic field in the direction perpendicular to the magnetic field being caused by the electric field. The transport of the dawn to dusk electric field crossing the plasmapause is plausible mechanism; the drift effects may be raised most effectively at the magnetic equator in the downward direction. Considering the distribution of the EPWAT phenomena, however, it is very likely that there may be the electric field component in radial direction towards or from the earth generating the circular drift motion in the equatorial disc. The drifting plasma across the magnetic field can easily generate the instabilities being coupled with the plasma in the plasmasphere. Precise studies are deferred for future to solve the problems relating to the mechanism of the 


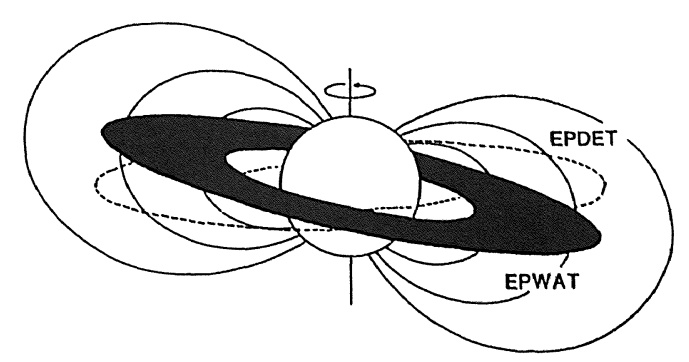

Fig. 9. The region of the occurrence of the EPWAT encircling the magnetic equator covering the altitude from $1000 \mathrm{~km}$ to $10,000 \mathrm{~km}$ (after OYA et al., 1991).

EPWAT, especially relating to the possible existence of the electric fields in the plasmasphere in the magnetic equator.

\section{Plasmasphere Structure}

After the discovery of the plasmapause by CARPENTER $(1963,1966)$, the concept of the plasmasphere has been established and plasmapause has been understood as the boundary region which is formed between the co-rotation plasma region and the magnetospheric convection region where plasma escapes by the magnetospheric convection (NISHIDA, 1966; BRICE, 1967).

The plasma distribution in the lower regions of the plasmasphere has been initially studied by ANGERAMI and THOMAS (1964) and by EVIATOR et al. (1964) and ANGERAMI (1966) also studied for the trough region. The model has been applied with a modification (AIKYO and ONDOH, 1971) to fit with the plasmapause formation. Observation results of the EXOS-D satellite have indicated that the feature of the wide variety of the plasma distributions which are not able to be simply understood in the frame of the previously established theories and concepts of the plasmapause.

\subsection{Disruption of the plasmapause}

Along the path of the (EXOS-D) satellite, sometimes, the feature of the plasma distribution shows clear existence of the plasmapause. In Fig. 10, a typical example of the plasmapause when the satellite traverses crossing the relatively lower altitude range has been shown. Though the satellite is in the altitude of $6000 \mathrm{~km}$, we can expand the observed data of plasmapause structure at the level corresponding to the magnetic equator. Very surprising changes take place in the time of the magnetic storms. To see this storm time variation we have selected here the storm period from Oct. 20 to Oct. 23, in 1989, where the largest Dst value of about $300 \mathrm{nT}$ had taken place at 16:00 UT Oct. 21, 1989. As has been given in Fig. 11 , there are no remarkable structure of plasmapause in the time of the main phase of the magnetic storm. It was believed from the traditional convection theory that the plasmapause is formed as the result of the plasma convection that makes differential flow between the corotating plasma in the plasmasphere. The theory requires, consequently that the plasmapause can be formed inner side with sharper edge of the density variation with 


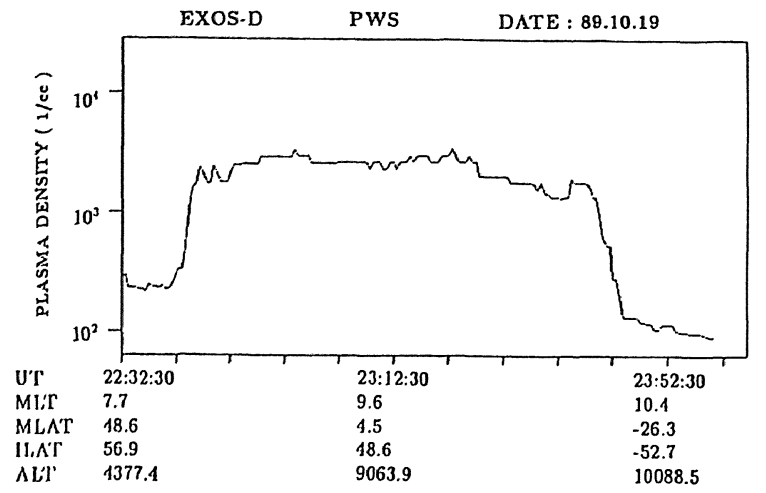

Fig. 10. Typical example of the plasmapause crossing observed on Oct. 19, 1989 when the Dst value is -28 to -32 . The plasma density is obtained from the upper hybrid mode wave frequency.

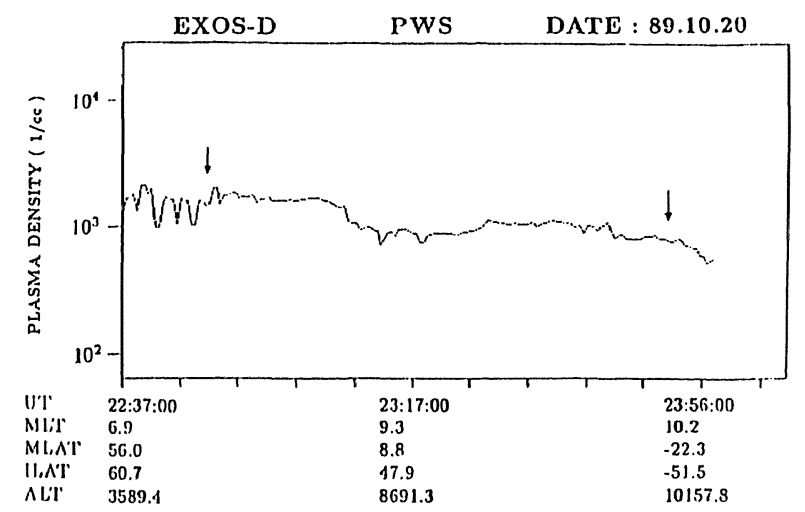

Fig. 11. The plasma density profile in the case of the main phase of the large magnetic storm on Oct. 20, 1989. No remarkable plasmapause structure can be recognized in the data from 22:35 UT to 24:00 UT though the satellite passed through the region of the plasmapause before the magnetic storm. Two arrows indicate the corresponding positions of the plasmapause before the destruction due to the start of the magnetic storm.

increasing convection velocity associated with the enhancement of the magnetic storms. Contrary to this prediction, the plasmapause observed by PWS on board the EXOS-D satellite shows complete disruption of the plasmapause.

The features of the disruption of the plasmapause have also been confirmed in global range of the plasmasphere of the large magnetic storms. In Fig. 12, where the plasma distribution calculated from the modified diffusive equilibrium theory (OYA and IIZIMA, 1991) has been given for the case of Oct. 19 and 20, in 1989 magnetic storm. 


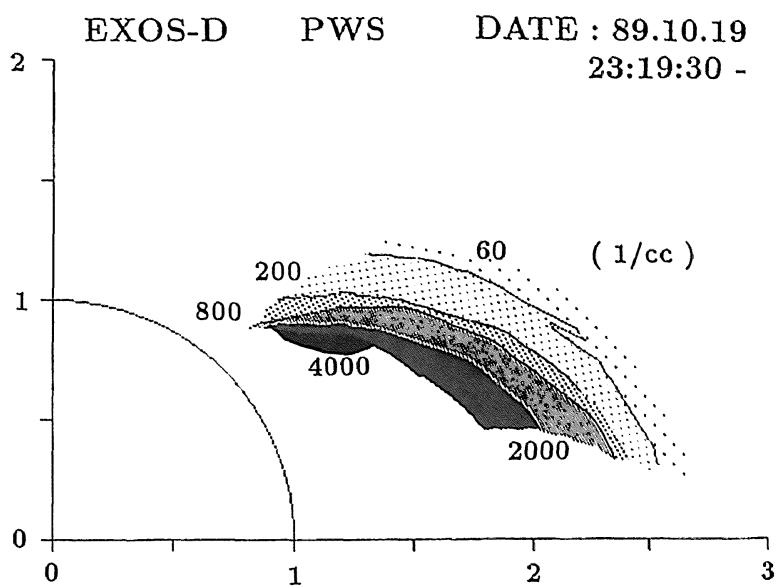

(a)

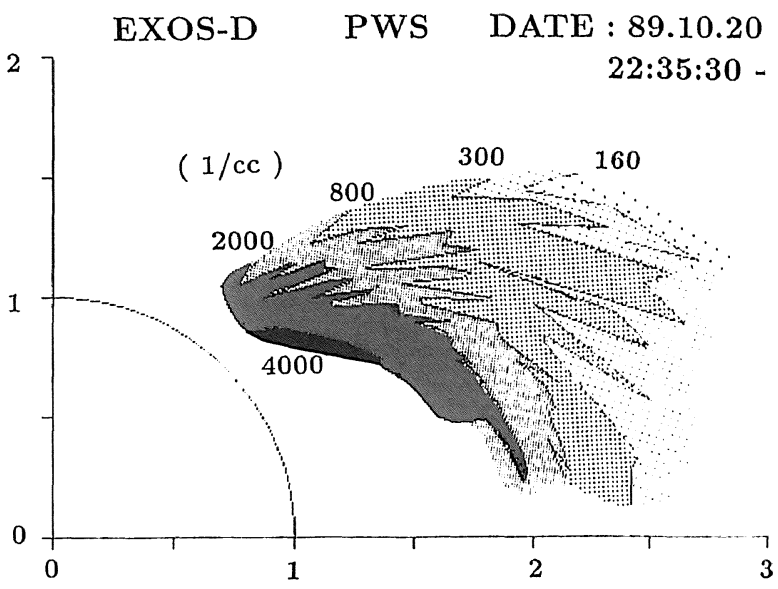

(b)

Fig. 12. Distribution of the plasma in the plasmasphere calculated by the modified diffusive equilibrium theory starting with the data observed at the EXOS-D satellite position. The number density values are shown with iso-density contours for a) pre-storm time, b) mainphase.

\subsection{Ring current effects}

In recovery phase of the magnetic storm, there are peculiar variation of the density distribution of the plasmasphere as has been given in Fig. 13a. This plasmaspheric plasma distribution is characterized by the existence of the two enhanced plasma density maxima located in the middle latitude with density depression in the low latitude region centered around the magnetic equator. Looking the shape of the plasma density distribution we call here the feature donkey ears. When this density variation is traced time by time through the main phase of the magnetic storm, the density depression regions are currently in the equatorial region where the upper hybrid mode wave frequency shows rather constant value 


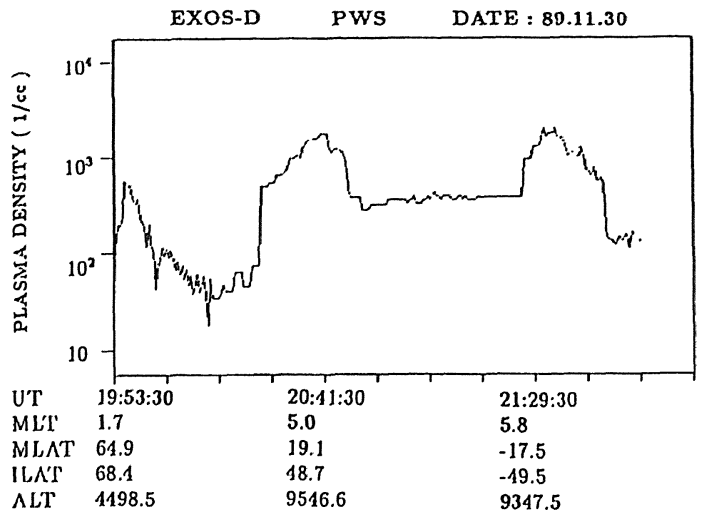

Fig. 13(a). The plasma density distribution along the satellite path in the recovery phase of the large magnetic storm which started 18 UT Nov. 27, 1989. Observation has been made from 19:53:30 UT to around 21:00:00 UT, Nov. 30, 1989.

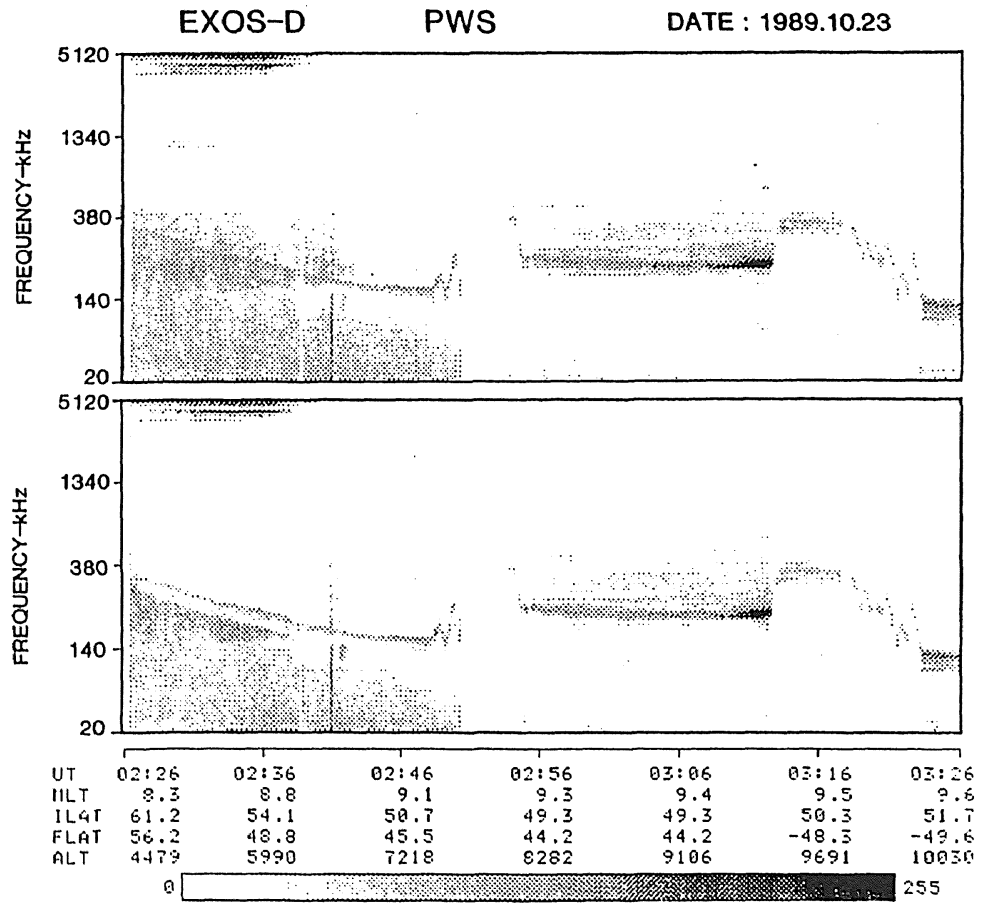

Fig. 13(b). Dynamic spectra of PWS in the same format with Fig. 7. Observation had been made from 02:26 UT to 03:26 UT, on Oct. 23, 1989. Between two maxima at 20:04:30 UT and at 21:33:30 UT, there is a region of the depressed plasma density with relatively constant and steady plasma distribution centered around the magnetic equator where the existence of the ring current was inferred from the magnetic field intensity that largely exceeds the IGRF model. 
though the satellite changes the position (see Fig. 13b). Considering the variation of the magnetic field intensity deduced from observed electron cyclotron frequency around this equatorial region, we can conclude that the observations were made inside of the current sheet where the magnetic field intensity increases from the value given by IGRF in the large magnetic storms. In the recovery phase of the magnetic storm, therefore, the plasma possibly starts to fill the region of the ring current where the plasma is confined by the diamagnetic balance conditions and making compression due to the retraction action of the expanded magnetic field towards the dipole type field. When the satellite makes crossing of the enhanced plasma regions as has been indicated in Fig. 14, we can see the two density enhanced regions making the two maxima of the plasma density distribution.

\subsection{Large turbulence in the plasmasphere}

In the last period of the recovery phase of the magnetic storms, the plasma density distribution inside the plasmapause shows the large turbulent feature as given in Fig. 15, where the density distribution is characterized by large oscillations. Interesting point is that the large oscillations of the plasma density appear in the region where the ring current regions are removed.

The complete theory to understand whole procedure of the storm time plasmasphere is not yet been presented. The EXOS-D results may provide important data for this direction of the studies to establish the real theory of the dynamics that is controlling the plasmapause and plasmasphere structures.

i)

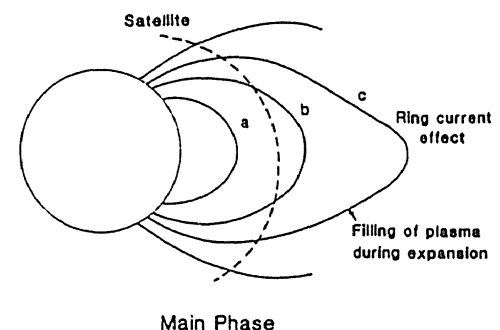

ii)

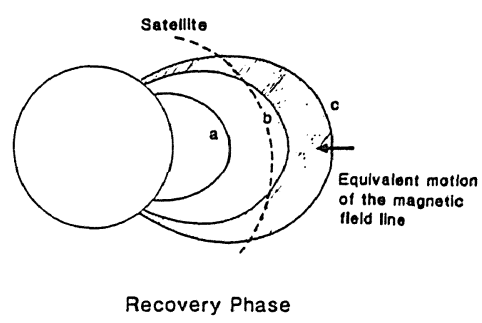

Fig. 14. A model to make interpretation of the compression processes of the plasmasphere in the recovery phase where the region of the strong ring current is removed. i) The magnetic field is largely expanded due to the distortion caused by intense ring current. ii) During recovery phase, the largely expanded magnetic field starts to move to return to the dipole magnetic field by decreasing ring current. The plasma in the flux tube is largely compressed due to retro-action of the magnetic field to return to the dipole magnetic field. 


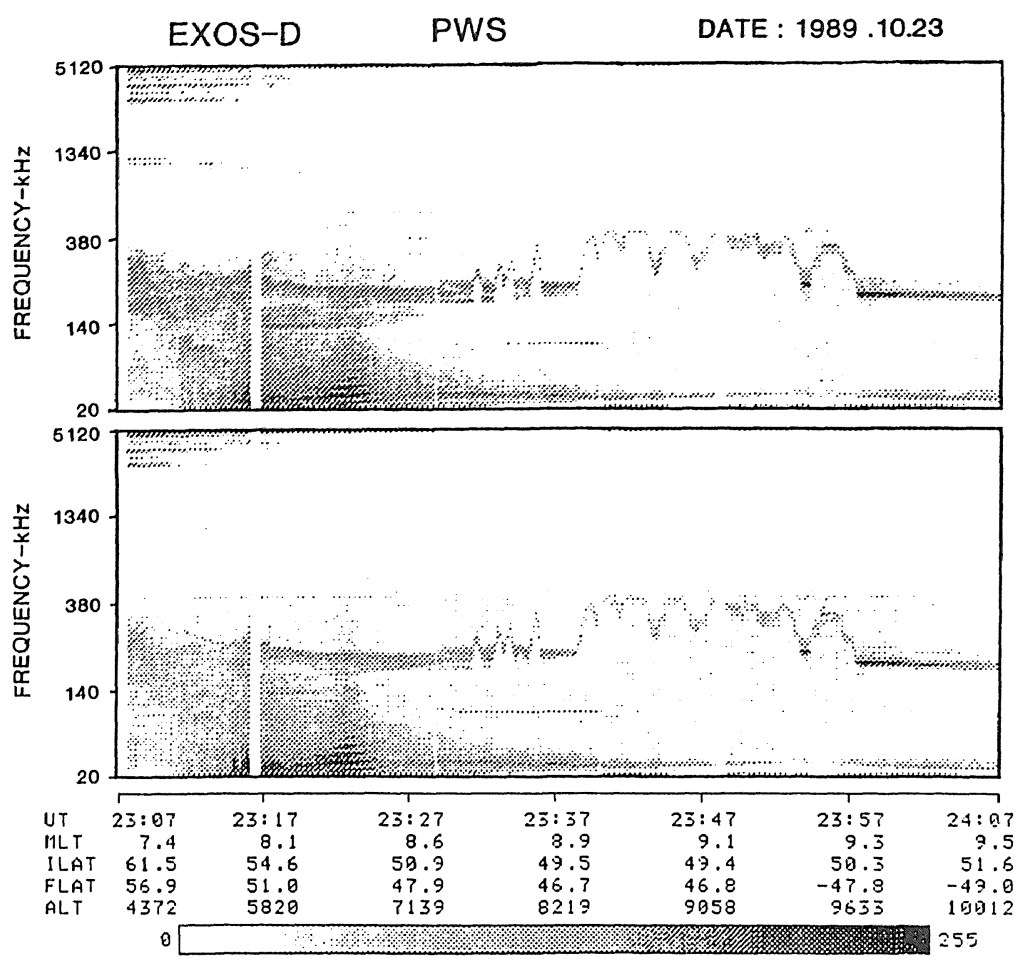

Fig. 15. Large turbulence of the plasmasphere indicated by large oscillation of the upper hybrid wave frequency. The dynamic spectrum is shown with same format with Fig. 7, for the observation of the EXOS-D satellite made from 23:07 UT to 24:07 UT on Oct. 23, 1989.

\section{Conclusion}

After the successful launching of the EXOS-D (Akebono) satellite, on Feb. 22, 1989, Plasma Wave and Sounder Experiments (PWS) has been providing data with high frequency resolution $(1 \mathrm{kHz})$ for the wave spectra from $20 \mathrm{kHz}$ to $5 \mathrm{MHz}$, time resolution of $2 \mathrm{sec}$ and with threshold of $0.1 \mu$ Volt $/ \mathrm{m}$. The results are not only restricted to the wave phenomena but the contribution is widely expanded to the related effects such as the particle precipitation and the free energy input which is generating the upper hybrid mode waves, $(n+1 / 2) f_{\mathrm{c}}$ emissions and also broad band noises. The plasma density distribution profiles deduced from the upper hybrid mode waves are also providing the data of the plasmasphere indicating the largely disturbed features of the plasmaspheric plasma density which has never been identified in the previous observations.

Wave phenomena in wide variety of the modes are observed:

1) Solar type III radio burst,

2) Auroral kilometric radiation (AKR),

3) Banded Rising and Falling LF waves (BRIFLE), 
4) Discrete HF emissions,

5) Terrestrial Hectometric Radiation (THR),

6) Upper hybrid mode waves,

7) Z-mode waves,

8) Quasi electrostatic whistler mode waves,

9) Plasmaspheric odd harmonic $(n+1 / 2) f_{\mathrm{c}}$ emissions, and

10) Broad band electrostatic noises.

AKR is important indicator of the acceleration regions providing the position, motion and range of the distributed acceleration regions. For the detail of the generation mechanism of AKR, the theory has not yet been settled whether cyclotron maser mechanism is operating or the Doppler conversion of the Z-mode waves which are converted from the upper hybrid mode waves is major processes. The PWS data are providing important keys with plasma parameters of AKR sources, such as the plasma density profiles, polarization, and Poynting flux data together with these source conditions. The results show that there are many cases indicating plasma conditions which are consistent with the conversion of Z-mode waves generated by the conversion of the upper hybrid mode waves. Among the plasma waves observed by the EXOS-D satellite, BRIFLE, discrete HF emissions, and plasmaspheric odd harmonic, $(n+1 / 2) f_{\mathrm{c}}$ emissions are new findings achieved by the PWS observations of EXOSD. Even for the previously identified subjects there are plenty of newly identified evidences based on the EXOS-D PWS data, for all of the other mode of above listed plasma waves; especially, it is important that the Z-mode waves are always existing associated with the upper hybrid mode waves which also exist in whole range of the plasmasphere in the altitude range higher than $1000 \mathrm{~km}$.

The upper hybrid mode waves show remarkable enhancements at the magnetic equator. A review has been made for this equatorial enhancements of the upper hybrid mode waves (OYA et al., 1990). The enhancements take place at the magnetic equator circulating around the entire plasmasphere ranging from the altitude of $1000 \mathrm{~km}$ to $10,000 \mathrm{~km}$ (upper limit of the orbit); the enhancements of the turbulent state of plasma circulating entire plasmasphere show the existence of the free energy input in the corresponding regions. The most possible cause of the origin of this free energy input is related to the radial electric field in the equatorial plane which generates the drift motion of the plasma at the magnetic equator. A candidate of this radial electric field is the potential difference between the earth and the magnetosphere.

From the upper hybrid mode waves, the accurate electron density profiles of the plasmasphere are deduced. The density profiles have revealed that the plasmapause is completely disrupted in the time of the large magnetic storms with decreasing electron density and the smoothed gradient of density distribution instead of the sharp edge of the density profile which is usually thought as the typical plasmapause structure. In the main phase of the large magnetic storm, the plasmaspheric odd harmonic, $(n+1 / 2) f_{\mathrm{c}}$ emissions are observed; the phenomena are not observed in the regular state of the magnetic conditions even the $\sum K p$ value is fairly large. The plasmaspheric odd harmonic phenomena suggest that plasma velocity distributions are largely distorted from the Maxwellian distribution, especially the energy of the plasma is enhanced in the direction perpendicular to the magnetic field. The phenomena also suggest that the large energy is injected from the magnetosphere into the plasmasphere.

In the recovery phase of large magnetic storms, wide variety of the plasma turbulences revealed in the plasmasphere. While the large sharp depression of the plasma density durates 
close to the position of the ring currents, there take place the local increments of the plasma density in the higher latitude ranges showing very unusual concave type plasma density distribution. That is, two maxima appear in north and south hemisphere of the plasmasphere, and we call the feature of the plasma density distribution donkey ears type distribution, here. In the phase of progress of the recovery of the magnetic storm, the plasmasphere starts to make retract from expanded feature to the dipole magnetic field controlled feature. During this phase of the contraction, the plasmasphere shows extremely large compression corresponding to the ring current position flux; and in further progress stage the plasma density distribution shows highly irregular feature. The density variation shows the change of 2-5 times increments in terms of the ratio of the maximum value to the minimum value.

The PWS data are thus providing important results not only for the studies on pure wave particle interaction processes but also for the studies on the dynamics of the plasmasphere. The present EXOS-D satellite observation will contribute to understand the whole feature of the energy input and corresponding dynamics of plasmasphere related to the case of extremely large irregularities of the plasma distribution.

The present work is directly depending on the EXOS-D (Akebono) observation. The author is grateful for ISAS, EXOS-D observation teams; and the author is especially indebted to PWS team member, Drs. A. Morioka, M. Iizima, K. Kobayashi, T. Ono, H. Miyaoka, and T. Obara. The data analyses are supported by STEP program by the ministry of education and culture, Japan.

\section{REFERENCES}

Aikyo, K. and T. ONDOH, Propagation of nonducted VLF waves in the vicinity of the plasmapause, J. Radio Res. Labs., 18, 153-182, 1971.

Alexander, J. K., H. H. Maeitson, and R. G. Stone, Type III radio bursts in the outer corona, Solar Phys., 8, 388-397, 1969.

Alvarez, H., F. Haddock, and R. P. Lin, Evidence for electron excitation of type III radio burst emission, Solar Phys., 26, 468-473, 1972.

Alvarez, H., R. P. LiN, and S. J. BAme, Fast solar electrons, interplanetary plasma and km-wave type III radio burst observed from IMP-6 spacecraft, Solar Phys., 44, 485-501, 1975.

Angerami, J. J. and J. O. Thomas, Studies of planetary atmospheres: 1. The distribution of electrons and ions in the earth's exosphere, J. Geophys. Res., 69, 4537-4560, 1964.

Angerami, J. J., A whistler study of the distribution of thermal electrons in the magnetosphere, Tech. Rep. SU-SEL-66-017, Radiosci. Lab., Stanford Electronics Labs., 1966.

Bahinsen, A., M. Jespersen, E. Ungstrup, and I. B. Iversen, Auroral hiss and kilometric radiation measured from the Viking satellite, Geophys. Res. Lett., 14, 471-474, 1987.

Bauer, S. J. and R. G. Stone, Satellite observations of radio noise in the magnetosphere, Nature, 218, 1145-1147, 1968.

Benediktov, E. A., G. G. Getmanstev, Yu. A. Sazonov, and A. F. Tarasov, Preliminary results of measurements of the intensity of distributed extraterresrial radio frequency emission at 725 and 1525 $\mathrm{kHz}$ frequency by the satellite electron-2, Kosm. Issled., 3, 614-617, 1965.

Benson, R. F., Source mechanism for terrestrial kilometric radiation, Geophys. Res. Lett., 2, 52-55, 1975.

Benson, R. F. and W. Calvert, ISIS 1 observations at the source of auroral kilometric radiation, Geophys. Res. Lett., 6, 479-485, 1979.

Benson, R. F., W. Calvert, and D. M. Klumpar, Simultaneous wave and Particle observation in the auroral kilometric radiation source region, Geophys. Res. Lett., 7, 959-1062, 1980.

Brice, N. M., Bulk motion of the magnetosphere, J. Geophys. Res., 72, 5193-5211, 1967.

Calvert, W., A Feedback model for the source of auroral kilometric radiation, J. Geophys. Res., 87, 8199-8214, 1982. 
CARPENTER, D. L., Whistler evidence of a "knee" in the magnetospheric ionization density profile, $J$. Geophys. Res., 68, 1675-1682, 1963.

CARPEnTER, D. L., Whistler studies of the plasmapause in the magnetosphere, 1, Temporal variations in the position of the knee and some evidence on plasma motions near the knee, J. Geophys. Res., 71, 693-710, 1966.

Dunkel, N., B. Ficklin, L. Rorden, and R. A. Helliwell, Low frequency noise observed in distant magnetosphere with OGO 1, J. Geophys. Res., 75, 1854-1862, 1970.

DUSENBERY, P. B. and L. R. LYONS, Generation of $Z$ mode radiation by diffuse auroral electron precipitation, J. Geophys. Res., 90, 2915-2920, 1985.

Evans, L. G., J. Fainberg, and R. G. Stone, A comparison of type III solar radio bursts theories using satellite radio observations and particle measurements, Solar Phys., 2l, 198-203, 1971.

Evans, L. G., J. FAINBERG, and R. G. STONE, Characteristics of type III exciters derived from low frequency radio observation, Solar Phys., 31, 501-511, 1973.

Eviator, A., A. M. Lenchek, and S. F. Singer, Distribution of density in an ion-exosphere of a nonrotating planet, Phys. Fluids, 7, 1775-1779, 1964.

Frank, L. A. and D. A. GuRNETT, Direct observations of low-energy solar electrons associated with a type III solar radio burst, Solar Phys., 27, 446-465, 1972.

Frederics, R. W., Plasma instability at $(n+1 / 2) f_{c}$ and its relationship to some satellite observations, $J$. Geophys. Res., 76, 5344-5348, 1971.

Gallagher, D. L. and D. A. Gurnett, Auroral kilometric radiation: Time-averaged source location, $J$. Geophys. Res., 84, 6501-6509, 1979.

GraedeL, T. E., The association of solar optical flares with type III solar bursts from 4 to $2 \mathrm{MHz}$ observed by OGO-III, Astrophys. J., 160, 301-308, 1970.

Green, J. L., D. A. GuRnett, and S. D. Shawhan, The angular distribution of auroral kilometric radiation, J. Geophys. Res., 82, 1825-1838, 1977.

Gregory, P. C., Radio emission from auroral electrons, Nature, 221, 350-352, 1969.

GuRnett, D. A., The earth as a radio source: Terrestrial kilometric radiation, J. Geophys. Res., 79, 42274238, 1974.

Gurnett, D. A., L. A. Frank, and R. P. Lepping, Plasma waves in distant magnetotail, J. Geophys. Res., 81, 6059-6071, 1976.

Gurnett, D. A., M. M. Baumback, and H. Rosenbauer, Stereoscopic direction finding analysis of a type III solar radio burst: evidence for emission at $2 f_{\mathrm{p}}, J$. Geophys. Res., 83, 616-622, 1978.

Harvey, C. C., Results from the UK-2 satellite, Ann. Astrophys, 28, 248, 1965.

Harvey, C. C., Radio emissions from geomagnetically trapped particles, Nature, 217, 50-51, 1968.

Hashimoto, K., K. YamaAshi, and I, Kimura, Three dimensional ray tracing of electrostatic cyclotron harmonic waves and $\mathrm{Z}$ mode electromagnetic waves in the magnetosphere, Radio Sci., 22, 579-594, 1987.

Hashimoto, K. and W. Calvert, Observation of the $\mathrm{Z}$ mode with DE-1 and its analysis by three dimensional ray tracing, J. Geophys. Res., 95, 3933-3942, 1990.

HoRne, R. B., Ray tracing of electrostatic waves in a hot plasma and its application to the generation of terrestrial myriametric radiation, Geophys. Res. Lett., 15, 553-556, 1988.

Horne, R. B., Path-integrated growth of electrostatic waves, J. Geophys. Res., 94, 8895-8909, 1989.

Huguenin, G. R., A. E. Lilley, W. H. McDonough, and M. D. Papagiannis, Measurements of radio noise at $0.700 \mathrm{Mc}$ and $2.200 \mathrm{Mc}$ from a high-altitude rocket probe, Planet. Space Sci., 12, 1157-1167, 1964.

JONES, D., Source of terrestrial nonthermal radiation, Nature, 260, 686-689, 1976.

Jones, D., G. Grapper, and R. Herring, Auroral kilometric radiation from field-aligned plasma density, Ann. Geophys., 2, 95-103, 1984.

KaISER, M. L., The solar elongation distribution of low-frequency radio bursts, Solar Phys., 45, 181-187, 1975.

KAISER, M. L. and R. G. STONE, Earth as an intense planetary radio source: Similarities to Jupiter and Saturn, Science, 189, 285, 1975.

Karpman, V. I., Ju. K. Alekhin, N. D. Borisov, and N. A. Ruabova, Electrostatic waves with frequencies above the gyrofrequency in a plasma with a loss-cone, Phys. Lett., Sect A, 44, 205-207, 1973.

Kennell, C. F., F. L. Scarf, R. W. Fredricks, J. H. McGeheE, and F. V. Coroniti, VlF electric-field observations in the magnetosphere, J. Geophys. Res., 75, 6136-6152, 1970. 
KIKUCHI, M., H. OYA, and A. MORIOKA, Generation processes of BRIFLE relating to the energetic particle precipitation near $L=1.2-1.6 .$, J. Geomag. Geoelectr. (Submitted), 1991.

KiKuChI, M., H. OYA, A. MORIOKA, K. Kobayashi, and M. IIZIMA, Ray path tracing of Banded Rising and Falling LF Emissions (BRIFLE) based on plasmasphere model deduced from the EXOS-D (AKEBONO) satellite result., SGEPSS spring meeting 1991: in abstract P. H11-05, 1991.

Kurth, W. S., M. M. BAumbaCK, and D. A. GurnetT, Direction-finding measurements of auroral kilometric radiation, J. Geophys. Res., 80, 2764-2770, 1975.

Kurth, W. S., J. D. Craven, L. A. Frank, and D. A. Gurnett, Intense electrostatic waves near the upper hybrid resonance frequency, J. Geophys. Res., 84, 4145-4164, 1979.

Kurth, W. S., D. A. Gurnetr, and R. R. Anderson, Escaping nonthermal continuum radiation, J. Geophys. Res., 86, 5519-5531, 1981.

LAASPERe, T., W. C. Johnson, and L. C. Semprebon, Observations of auroral hiss, LHR noise, and other phenomena in the frequency range $20 \mathrm{~Hz}$ to $540 \mathrm{kHz}$ on OGO 6, J. Geophys. Res., 76, 4477-4493, 1971.

LIM, T. L. and T. LAASPERE, An evaluation of Cerenkov radiation from auroral electrons with energies down to $100 \mathrm{eV}, J$. Geophys. Res., 77, 4145-4157, 1972.

LiN, C. S., H. K. Wong, J. KogA, and J. L. BuRCH, Excitation of low-frequency waves by auroral electron beams, J. Geophys. Res., 94, 1327-1337, 1989.

Lin, R. P., D. W. Potter, D. A. Gurnetr, and F. L. SCARF, Energetic electrons and plasma waves associated with a solar type III radio burst, Astrophys. J., 251, 364-373, 1981.

MAGGS, J. E., Theory of electromagnetic waves on auroral field lines, J. Geomag. Geoelectr., 30, 273$287,1978$.

MenietTI, J. D. and C. S. LiN, Ray tracing of Z-mode emissions from source regions in the high altitude auroral zone, Geophys. Res. Lett., 12, 385-388, 1985.

MIYAKE, W. and H. OYA, Direction-finding analysis of type III solar radio bursts observed by the Jikiken (EXOS-B) satellite, J. Geomag. Geoelectr., 41, 261-272, 1989.

Mohr, B., R. G. Stone, and H. H. Malitson, Structure of type III solar bursts at long wavelengths, Proceeding URSI, Washington, D.C., U.S.A. 54, 1969.

Morioka, A., H. OYA, and S. MiYATAKe, Terrestrial kilometric radiation observed by satellite Jikiken (EXOS-B), J. Geomag. Geoelectr., 33, 37-62, 1981.

Morioka, A., H. Oya, H. Miyaoka, T. Ono, T. Obara, H. Yamagishi, and H. Fukunishi, Wave-particle interaction in the auroral ionosphere in LF and HF range: Results from antarctic rocket observation, J. Geomag. Geoelectr., 40, 923-937, 1988.

Morioka, A., H. OYA, and K. Kobayashi, Polarization and mode identification of auroral kilometric radiation by PWS system on board the Akebono (EXOS-D) satellite, J. Geomag. Geoelectr., 42, 443$458,1990$.

Mosier, S. R., M. L. Kaiser, and L. W. Brown, Observations of noise bands associated with the upper hybrid resonance by IMP 6 radio astronomy experiment, J. Geophys. Res., 78, 1673-1679, 1973.

MULDREW, D. B., Preliminary results of ISIS 1 concerning electron-density variations, ionospheric resonances, and Cerenkov radiation, Space Res., 10, 786, 1970.

NishidA, A., Formation of Plasmapause, or magnetospheric plasma knee, by the combined action of magnetospheric convection and plasma escape from the tail, J. Geophys. Res., 71, 5669-5679, 1966.

Okuda, H., M. Ashour-Abdalla, M. S. Chance, and W. S. Kurth, Generation of nonthermal continuum radiation in the magnetosphere, J. Geophys. Res., 87, 10457-10462, 1982.

OMIDI, N., C. S. WU, and D. A. GuRnetT, Generation of auroral kilometric and Z-mode radiation by the cyclotron maser mechanism, J. Geophys. Res., 89, 883-895, 1984.

OMIDI, N. and D. A. GuRnetr, Growth rate calculations of auroral kilometric radiation using the relativistic resonance condition, J. Geophys. Res., 87, 2377-2383, 1982.

OMIDi, N. and D. A. GuRnett, Path-integrated growth of auroral kilometric radiation, J. Geophys. Res., 89, 10801-10812, 1984.

OMIDI, N. and C. S. WU, The effect of background plasma density on the growth of ordinary and Z-mode emissions in the auroral zone, J. Geophys. Res., 90, 6641-6646, 1985.

OYA, H., Sequence of diffuse plasma resonances observed on Alouette ionogram, J. Geophys. Res., 75, 4279-4285, 1970. 
OYA, H., Conversion of electrostatic waves into the electromagnetic waves: Numerical calculation of the dispersion relation for all wavelengths, Radio. Sci., 6, 1131-1141, 1971 a.

OYA, H., Verification of theory on weak turbulence relating to the sequence of diffuse plasma resonances in space, Phys. Fluids, 14, 2487-2499, $1971 \mathrm{~b}$.

OYA, H., Origin of Jovian decameter wave emissions-Conversion from the electron cyclotron plasma waves to the ordinary mode electromagnetic wave, Planet. Space Sci., 22, 687-708, 1974.

OYA, H. and A. MORIOKA, Observational evidence of $\mathrm{Z}$ and L-O mode waves as the origin of auroral kilometric radiation from Jikiken (EXOS-B) satellite, J. Geophys. Res., 88, 6189-6203, 1983.

OYA, H., A. MORIOKA, and T. OBARA, Leaked AKR and terrestrial hectometric radiations discovered by the plasma wave and planetary plasma sounder experiments on board the Ohzora (EXOS-C) satellite: Instrumentation and observation results of plasma wave phenomena, J. Geomag. Geoelectr., 37, 237-262, 1985.

Oya, H., A. Morioka, K. Kobayashi, M. Iizima, T. Ono, H. Miyaoka, T. OKada, and T. Obara, Plasma wave observation and sounder experiments (PWS) using the Akebono (EXOS-D) satellite-Instrumentation and initial results including discovery of the high altitude equatorial plasma turbulence, J. Geomag. Geoelectr, 42, 411-442, 1990.

OYA, H., Origin of Auroral kilometric radiation as conversion of the upper hybrid mode plasma waves, Proc. Japan Acad., 66, Ser B, 129-134, 1990.

OYA, H. and M. IIzIMA, Conversion mechanism of Z-mode waves as the source of auroral kilometric radiation by the relative plasma motion, submitted to Radio Sci., 1991.

OYA, H., M. IIZIMA, and A. MorioKA, Plasma turbulence disc circulating the equatorial region of the plasmasphere identified by the plasma wave detector (PWS) on board the Akebono (EXOS-D) Satellite, Geophys. Res. Lett., 18, 329-332, 1991.

OYA. H., M. KIKUCHI, and A. MORIOKA, Plasmaspheric LF radio wave generation and propagation observed by the EXOS-D satellite., J. Geomag. Geoelectr., 1991 (Submitted).

Taylor, W. W. and S. D. Shawhan, Cerenkov radiation and VLF Hiss, J. Geophys. Res., 79, 105-117, 1974.

Pottelette, R., M. Malingre, N. Dubouloz, B. Aparicio, R. Lundin, G. Holmgren, and G. Marklund, High-frequency waves in the cusp/cleft regions, J. Geophys. Res., 95, 5957-5971, 1990.

SAWYER, C. and J. W. WARWICK, Wide visibility of kilometric type III bursts, Astron. and Astrophys, 177, 277-282, 1987.

Stone, R. G., Radio physics of the outer solar system, Space Sci. Rev., 14, 534-551, 1973.

Voots, G. R., D. A. Gurnett, and S.-I. Akasofu, Auroral kilometric radiation as an indicator of auroral magnetic disturbances, J. Geophys. Res., 82, 2259-2266, 1977.

WALSH, D., F. T. HADDOCK, and H. F. SCHUlte, Cosmic radio intensities at 1.225 and 2.0 Mc measured up to an altitude of $1700 \mathrm{~km}$, Space Res., 4, 935, 1964.

Weber, R. R., R. J. Fitzenreiter, J. C. Novace, and J. Fainberg, Interplanetary baseline observations of type III solar radio bursts, 54, 431-439, 1977.

Young T. S. T., J. D. Callen, and J. E. MCCune, High frequency electrostatic waves in the magnetosphere, J. Geophys. Res., 78, 1082-1099, 1973.

Wu, C. S. and L. C. LEE, A theory of the terrestrial kilometric radiation, Astrophys. J., 230, 621-626, 1979. 